\title{
TWO PUMP OPA FOR OTDM PULSES AMPLIFICATION
}

Lucia Marazzi ${ }^{1}$, Paola Parolari ${ }^{1}$, Pierpaolo Boffi ${ }^{1}$, Elisabetta Rognoni ${ }^{1}$, Paolo Gaviraghi $^{1}$ and Mario Martinelli ${ }^{1,2}$

${ }^{1}$ CoreCom, via G. Colombo,81, 20134 Milano, Italy marazzi@corecom.it

${ }^{2}$ Politecnico di Milano, piazza L. da Vinci 32, 20133 Milano, Italy

\begin{abstract}
Necessity to exploit new bandwidth has moved research interest towards new amplification solution, which support the exploitation of the fiber bandwidth and of short optical pulses. 2P-OPA seems quite promising as they offer high project flexibility, high gain uniformity and low accumulated dispersion. We present and discuss a 2P-OPA realization and its behavior with $\mathrm{CW}$ and picosecond pulses.
\end{abstract}

\section{INTRODUCTION}

Exploitation of available bandwidth has moved towards two directions: accommodation of many channels at different wavelengths in Dense Wavelength Division Multiplexed (DWDM) systems or very high bit rate Optical Time Division Multiplexed (OTDM) systems. Nowadays this distinction may not be very significant as each WDM channel may be in fact a high bit rate OTDM channel, which may employ picosecond optical pulses. In this scenario the development of new amplification techniques, which support the exploitation of the fiber bandwidth and of short optical pulses, has gained a great interest. In particular amplification solutions based on nonlinear effects show good potentialities as they avoid the constraint of ions fixed energy levels and lifetimes. Both Optical Parametric Amplifiers (OPA's), which are based on fiber Four Wave Mixing (FWM), and Raman amplifiers present high project flexibility. In principle their bandwidth can be arbitrarily chosen provided correct pump wavelength and power. In particular broadband significant gain [1] and low noise figure [2] OPA's 
have been demonstrated. Moreover the Kerr effect is known to respond at less than $100 \mathrm{fs}$ and seems thus very interesting for picosecond OTDM pulses amplification.

Aim of this contribution is to experimentally analyze the impact of OPA's exploiting Dispersion Shifted (DS) fiber on OTDM pulses. We will concentrate on two-pump OPA (2P-OPA) solutions, which seem particularly suitable for OTDM pulses amplification. As can be seen in Fig.1a, in the three waves scheme (1POPA), the pump, close to the zero dispersion wavelength, transfers energy toward signal and idler, which are symmetrically located with respect to the zero dispersion wavelength. On the contrary in the 2P-OPA (fig. $1 \mathrm{~b}$ ) the two pumps are symmetric with respect to the DS fiber zero dispersion wavelength. Thus amplification region is not only very homogeneous, as can be seen in the inset of Fig.1, where simulation results are shown, but also shows very low dispersion. Both characteristics can better support picosecond pulse propagation, which is affected by dispersion and, due to broad picosecond pulse spectra, also by gain non-uniformities. Moreover total pump power in the two-pump scheme is divided into two pumps thus reducing Stimulated Brillouin Scattering (SBS) impact [3] with respect to the one pump solution.

a)

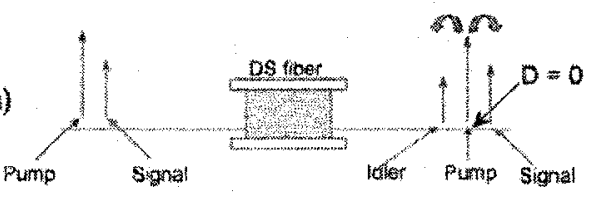

b)
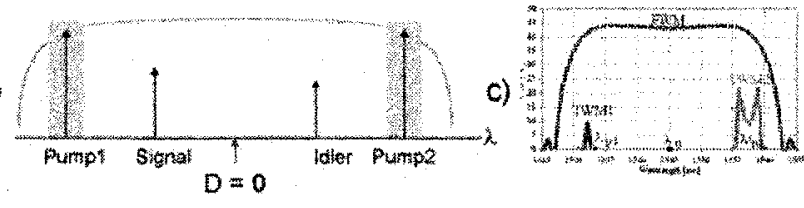

Figure 1. a)OPA e b)2P-OPA schemes. In c) 2P-OPA gain profile is shown together with the two TWM's gain regions. Simulation parameter are: fiber length $5 \mathrm{~km}$, $\lambda_{0}-\lambda_{c} \cong 0.2 \mathrm{~nm}, \lambda_{\text {pumpl }}=1532.25 \mathrm{~nm}, \lambda_{\text {pump } 2}=1557.57 \mathrm{~nm}, \mathrm{P}_{\text {pump } 1}=P_{\text {pump } 2}=0.5 \mathrm{~W}$.

\section{TWO PUMP-OPA PROJECT}

The influence of 2P-OPA project parameters such as pump power and spectral allocation on the gain profile of 2P-OPA have been investigated with a simplified model, which takes into account also pump depletion. We focused on main process, i.e. non-degenerate FWM: two photons, one from each pump, combine to generate one signal photon and one idler photon satisfying the relation 
$\omega_{p 1}+\omega_{p 2}=\omega_{3}+\omega_{i}$ where $\omega_{i}$ is idler pulsation. TWM1, which involves pumpl, the signal and the respective idler1, and TWM2, which involves pump2, the signal and the respective idler2, are separately considered. The analysis of the complete equations relating TWM's [5,6], taking into account also pump depletion, has evidenced that the same actions which advantage 2P-OPA gain uniformity, e.g. pump power increase and pump distance reduction, also advantage extensions of the TWM gain bandwidths [4]. Actually in order to have 2P-OPA uniform gains it is necessary to look for a trade-off between these two effects. A sample of the performed simulations is presented in Fig.1c and shows that this can partly be accomplished by unbalancing pump powers, giving a first project hint. This hint together with limits due to available devices and nonlinear media has been taken into account in the realization of a 2P-OPA, which has been tested for OTDM pulse amplification and will be discussed in the next section.

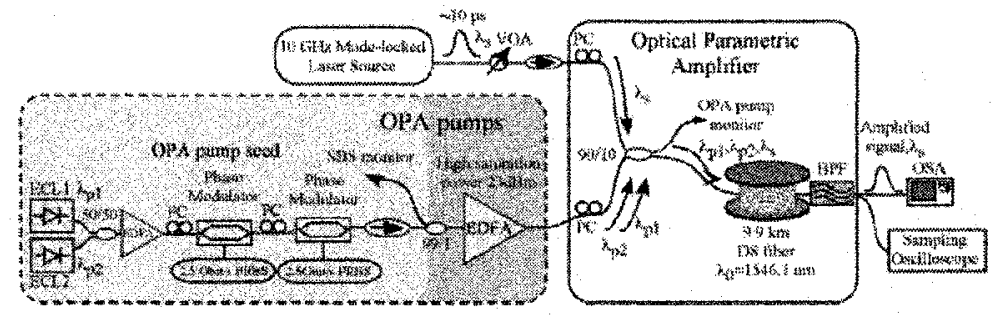

Figure 2. Set up.

\section{EXPERIMANTAL SET UP}

Experimental setup is shown in Fig.2. Nonlinear medium is a $9.9 \mathrm{~km}$ long DS fiber with zero dispersion wavelength of $1546.1 \mathrm{~nm}$ and dispersion slope of 0.062 $\mathrm{ps} /\left(\mathrm{nm}^{2} \mathrm{~km}\right)$ and nonlinear coefficient $\gamma=1.5 \mathrm{~km}^{-1} \mathrm{~W}^{-1}$. Pumps are generated by two ECL lasers coupled together and subsequently amplified and further fed into the nonlinear fiber through a 90/10 coupler. Pumps wavelengths and powers are respectively $\lambda_{\text {pump } 1}=1537.32 \mathrm{~nm}, \lambda_{\text {pump2 }}=1553.16 \mathrm{~nm} \mathrm{P} P_{\text {pump1 }}=14.11 \mathrm{dBm}$, $P_{\text {pump } 2}=13.5 \mathrm{dBm}$. Probe signal is a $10 \mathrm{GHz} 7$ ps mode-locked pulsed source by Pritel. Due to high powers involved two phase modulators, operated with two different 2.5Gbit/s PRBS sequences, broaden pump optical spectrum to suppress SBS insurgence. Relative state of polarization of the three interacting fields is controlled. Fig. 3 shows $\mathrm{CW}$ characterization of the projected and realized 2P-OPA. As spontaneous amplification (a) and small signal gain (b) show, with $14.84 \mathrm{~nm}$ pumps spacing, nearly $13 \mathrm{~nm}$ of uniform bandwidth are found. Uniformity is within $1 \mathrm{~dB}$. Signal power is $-17.2 \mathrm{dBm}$ and average on-off gain is $6.5 \mathrm{~dB}$. NF 
values are all higher than $20 \mathrm{~dB}$, which is an unacceptable value for a telecom amplifier, yet it has already been demonstrated [2] that by properly filtering pump noise contributions NF can be significantly reduced.

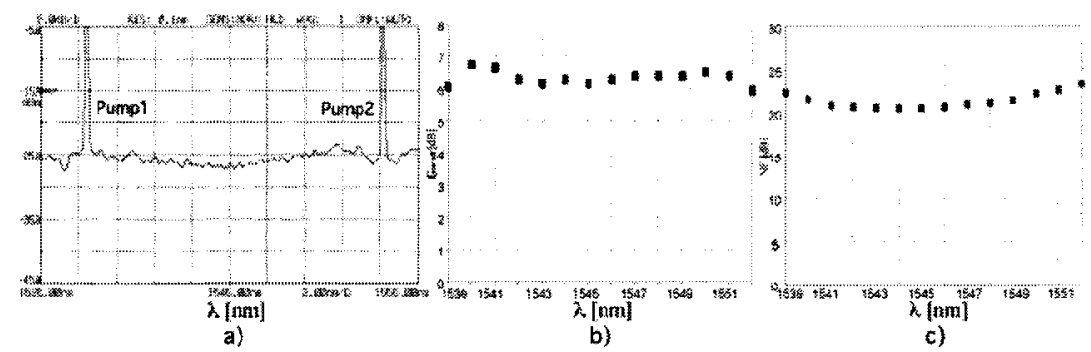

Figure 3. a) Spontaneous 2P-OPA parametric gain measured with optical spectrum analyzer, b) CW 2P-OPA gain c) measured 2P-OPA noise figure.

On-off gain for picosecond pulses has been measured as well. Results are plotted in Fig.4, compared with CW small signal gain. Slightly higher gain value are found for low power OTDM pulses than for $\mathrm{CW}$ signal: this is probably due to spectral peak wavelength gain measure. As expected this value saturates when peak pulse power is increased from $-3 \mathrm{dBm}$ to $11 \mathrm{dBm}$.

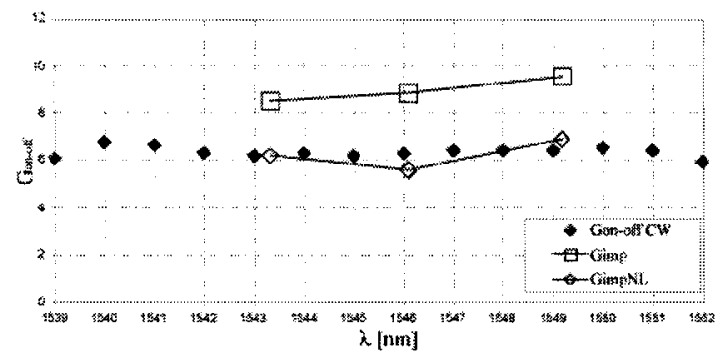

Figure 4. 2P-OPA on-off gain for $\mathrm{CW}$, low power pulsed and high power pulsed signal.

Final OTDM pulse amplification has been evaluated by means of pulse autocorrelator traces, to measure pulse broadening which results for OTDM signal in power penalty at the receiver. Employed set up is shown in Fig.5: an EDFA is needed in front of the autocorrelator and polarization controllers maximize second harmonic generation. We measured pulse broadening in the fiber without and with OPA amplification along the amplifier bandwidth. Fig.6 shows an example of the performed measure. OTDM pulse wavelength is $1546.1 \mathrm{~nm}$, which corresponds to the fiber zero dispersion wavelength. As expected, no broadening is observed between the pulse at the fiber input (Fig. 6a, first row) and at the fiber output (Fig. 
6b, first row), while when amplification is taken into account pulse broadens from $7.5 \mathrm{ps}$ to $7.79 \mathrm{ps}$ (3.9\%) (Fig. 6c, first row). All over the amplifier bandwidth we observed broadening ranging from $3.9 \%$ to $4.9 \%$ (close to the two pumps). Input pulse peak power is $-3 \mathrm{dBm}$. When input power increases to $11 \mathrm{dBm}$ observed broadening ranges from $2 \%$, as expected in the anomalous dispersion region, to $4 \%$ as can be see on the second row of Fig.6.

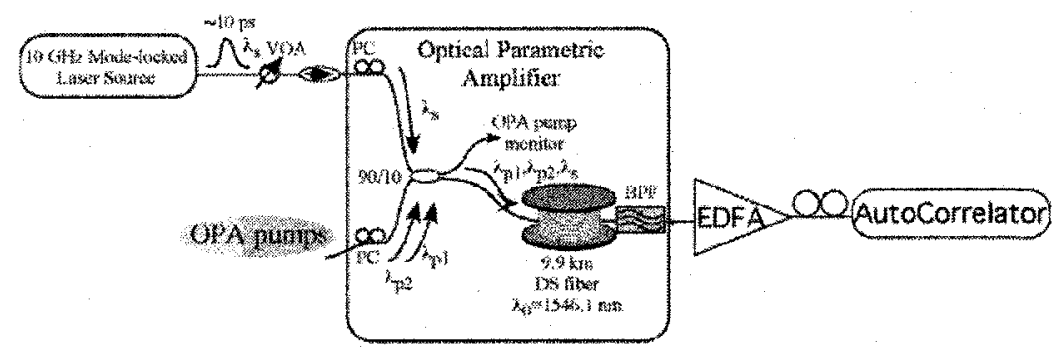

Figure 5. OTDM pulse broadening measure set up.
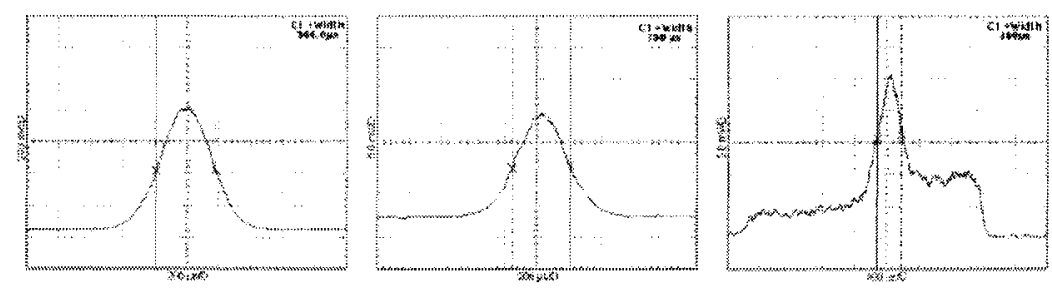

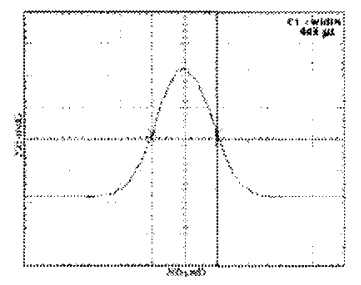

a)

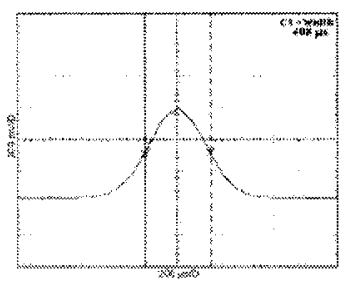

b)

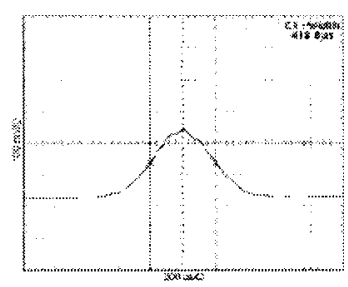

c)

Figure 6. Pulse autocorrelation traces at the fiber input (a), at the fiber output without parametric amplification (b), at the $2 \mathrm{P}-\mathrm{OPA}$ output (c). Input signal peak power $-3 \mathrm{dBm}$ (first row), $11 \mathrm{dBm}$ (second row). 


\section{CONCLUSIONS}

In conclusion we analyzed the 2P-OPA behavior as a possible solution to the necessity of exploiting new optical bandwidth when dealing both with WDM and high bit rate OTDM systems. OPA-2P has shown many advantages as high flexibility of project and gain uniformity over significant bandwidth. We projected and realized a $2 \mathrm{P}-\mathrm{OPA}$, which has been fully characterized for $\mathrm{CW}$ signals. We tested also the amplifier with $10 \mathrm{GHz} 7 \mathrm{ps}$ OTDM pulses. Measured broadenings over the amplification bandwidth are all within $4-5 \%$. These values do not comport strong impairments on OTDM signal even at very high bit rate.

\section{ACKNOWLEDGMENTS}

This work was supported by the MIUR project "Studio e sperimentazione di architetture in fibra per l'amplificazione ottica di segnali OTDM" under Project 2001098217004.

\section{REFERENCES}

[1] M.E. Marhic, Y. Park, "Broadband fiber-optical parametric amplifier and wavelength converters with low-ripple Chebyshev gain spectra", Opt. Lett., vol. 21, n. 17, pp. 1354$1356,1996$.

[2] L. Marazzi, P. Parolari, S. Seghizzi, M. Martinelli "Raman-generated pump impact on optical parametric amplification" IEEE Photon. Technol. Lett., 16, 1, pp.78-80, 2004.

[3] C.J. McKinstrie, S. Radic, "Parametric amplifiers driven by two pump wave with dissimilar frequencies", Opt. Lett., vol. 27, n. 13, pp. 1138-1140, 2002.

[4] C.J. McKinstrie, S. Radic, R. Chraplyvy, "Parametric Amplifier Driven by Two Pump Waves", J. Sel. Top. Quantum Electron., 8, 3, pp. 538-547, 2002.

[5] J. Hansryd, P.A. Andrekson, "Fiber-Based Optical Parametric Amplifiers and Their Applications", J. Sel. Top. Quantum Electron., vol. 8, n. 3, pp. 506-518, 2002.

[6] K. Inoue, T. Mukai, "Signal wavelength dependence of gain saturation in a fiber optical parametric amplifier", Optics Letters, vol. 26, n. 1, pp. 10-12, 2001. 